\title{
The antimicrobial effects of Citrus limonum and Citrus aurantium essential oils on multi-species biofilms
}

Sarah Almeida Coelho Oliveira(a) Jéssica Rabelo Mina Zambrana(a) Fernanda Bispo Reis Di lorio(b) Cristiane Aparecida Pereira ${ }^{(a)}$ Antonio Olavo Cardoso Jorge ${ }^{(a)}$

(a) Department of Biosciences and Oral Diagnosis, Institute of Science and Technology, Universidade Estadual Paulista - UNESP, São José dos Campos, SP, Brazil.

(b) Department of Earth and Exact Sciences, Laboratory of Hybrid Materials, Institute of Environmental, Chemical and Pharmaceutics Sciences, Universidade Federal de São Paulo - Unifesp, São Paulo, SP, Brazil.

Declaration of Interests: The authors certify that they have no commercial or associative interest that represents a conflict of interest in connection with the manuscript.

\section{Corresponding author:}

Antonio Olavo Cardoso Jorge

E-mail: olavojorge@uol.com.br

htrp://dx.doi.org/10.1590/S1806-83242013005000024 Epub Oct 07, 2013

Submitted: May 29, 2013

Accepted for publication: Jul 31, 2013

Last revision: Aug 18, 2013
Abstract: The aim of this study was to evaluate the effects of Citrus limonum and Citrus aurantium essential oils (EOs) compared to $0.2 \%$ chlorhexidine $(\mathrm{CHX})$ and $1 \%$ sodium hypochlorite $(\mathrm{NaOCl})$ on multispecies biofilms formed by Candida albicans, Enterococcus faecalis and Escherichia coli. The biofilms were grown in acrylic disks immersed in broth, inoculated with microbial suspension $\left(10^{6}\right.$ cells $\left./ \mathrm{mL}\right)$ and incubated at $37^{\circ} \mathrm{C} / 48 \mathrm{~h}$. After the biofilms were formed, they were exposed for 5 minutes to the solutions $(\mathrm{n}=10)$ : C. aurantium $\mathrm{EO}$, C. limonum EO, $0.2 \% \mathrm{CHX}, 1 \% \mathrm{NaOCl}$ or sterile saline solution $[0.9 \%$ sodium chloride $(\mathrm{NaCl})]$. Next, the discs were placed in sterile $0.9 \% \mathrm{NaCl}$ and sonicated to disperse the biofilms. Tenfold serial dilutions were performed and the aliquots were seeded onto selective agar and incubated at $37^{\circ} \mathrm{C} / 48 \mathrm{~h}$. Next, the number of colony-forming units per milliliter was counted and analyzed statistically (Tukey test, $p \leq 0.05$ ). C. aurantium $\mathrm{EO}$ and $\mathrm{NaO}-$ $\mathrm{Cl}$ inhibited the growth of all microorganisms in multi-species biofilms. C. limonum EO promoted a $100 \%$ reduction of C. albicans and E. coli, and $49.3 \%$ of E. faecalis. CHX was less effective against C. albicans and E. coli, yielding a reduction of $68.8 \%$ and $86.7 \%$, respectively. However, the reduction of E. faecalis using $\mathrm{CHX}(81.7 \%)$ was greater than that obtained using C. limonum EO. Both Citrus limonum and Citrus aurantium EOs are effective in controlling multi-species biofilms; the microbial reductions achieved by EOs were not only similar to those of $\mathrm{NaOCl}$, but even higher than those achieved by CHX, in some cases.

Descriptors: Biofilms; Citrus limonum; Citrus aurantium.

\section{Introduction}

The oral cavity is heavily colonized by a complex, relatively specific and highly interrelated group of microorganisms that are organized in biofilms. Microbial biofilms are composed of microorganisms that adhere to each other and/or to surfaces or interfaces and are embedded in an extracellular polymeric matrix, which includes water and nutrient channels. ${ }^{1}$

Candida albicans is a commensal yeast from the oral cavity and is the most virulent species of the genus. It is the main cause of oral candidiasis. ${ }^{2}$ Escherichia coli is a transient colonizer of the oral cavity, and the endotoxin produced by this bacterium plays an important role in the onset and perpetuation of periapical lesions and inflammatory bone resorption. ${ }^{3}$ Enterococcus faecalis is a normal inhabitant of the oral cavity 
and is associated with different forms of periradicular disease, including primary endodontic infections and persistent infections. ${ }^{4,5}$

Mouth rinses are used as adjuncts to mechanical oral hygiene. The use of mechanical control alone to reduce recalcitrant biofilms in the oral cavity has been challenged, because it is considered timeconsuming and, most importantly, insufficient for promoting effective oral hygiene. ${ }^{1,6}$ An increasing number of innovative formulation technologies have called for more predictive laboratory models to assess the preclinical biocidal efficacy of mouth rinses. Because biofilm microorganisms may be $10-1000$ times more resistant to antimicrobial agents than planktonic cells of the same species, ${ }^{7}$ biofilm tests may be a more predictive assessment of mouth rinse efficacy than tests with free-living cells.

Medicinal plants may be explored to produce valuable herbal products, which are frequently used as natural alternatives to treat several diseases. ${ }^{8}$ The antimicrobial components of these plants can inhibit bacterial and fungal growth through mechanisms that are distinct from those used by ordinary antibiotics, underscoring their clinical significance. ${ }^{9}$

Citric and acidic fruits contain healthy and nutritive contents. The peels of Citrus fruits are rich in flavonoids, especially many polymethoxylated flavones, which are very rare in other plants. ${ }^{10}$ The antimicrobial activities of several Citrus species, namely, C. aurantium, C. bergamia, C. limonum, C. maxima, C. paradisi, and C. reticulata, have been investigated, but the related tests have typically involved microorganisms in planktonic cultures, ${ }^{11-14}$ and few studies have been conducted on microbial biofilms. ${ }^{15,16}$

The aim of this study was to evaluate the effects of C. limonum (lemon) and C. aurantium (bergamot) essential oils (EOs) compared to $0.2 \%$ chlorhexidine $(\mathrm{CHX})$ and $1 \%$ sodium hypochlorite $(\mathrm{NaOCl})$ solutions on multi-species biofilms formed on acrylic resin by Candida albicans, Enterococcus faecalis and Escherichia coli reference strains.

\section{Methodology Microorganisms}

Three reference strains [American Type Culture Collection (ATCC), C. albicans (ATCC 18804), E. coli (ATCC 25922) and E. faecalis (ATCC 29212)] were used in the study.

Standard suspensions of each strain with optical densities equivalent to $10^{6}$ cells $/ \mathrm{mL}$ were prepared by seeding the strains in Sabouraud agar (Difco, Detroit, USA) for C. albicans, or in brain heart infusion (BHI) agar (Difco, Detroit, USA) for E. coli and E. faecalis, and incubated at $37^{\circ} \mathrm{C}$ for $24 \mathrm{~h}$.

After incubation, the cells were suspended a second time in sterile saline solution [0.9\% sodium chloride $(\mathrm{NaCl})]$, and the number of cells in the suspension was counted in a spectrophotometer (B582, Micronal, São Paulo, Brazil). The optical density and wavelength parameters used were 0.284 and $530 \mathrm{~nm}$ for C. albicans; 0.324 and $590 \mathrm{~nm}$ for $E$. coli; and 0.298 and $760 \mathrm{~nm}$ for E. faecalis.

\section{Evaluation of EOs and other chemical solutions}

EOs were collected from air-dried peels of C. aurantium (bergamot) and C. limonum (lemon) fruits. The peels were placed in a round-bottom distillation flask to which distilled water was added. The EOs were obtained by hydrodistillation for $3 \mathrm{~h}$ with the Clevenger apparatus. The oils were separated, dried over anhydrous sodium sulfate, and stored in an amber bottle at $4^{\circ} \mathrm{C}$ until use. ${ }^{17}$

The antimicrobial effects of the EOs were also compared to those of $0.2 \% \mathrm{CHX}$ and $1 \% \mathrm{NaOCl}$ (Byoformula, São Paulo, Brazil). The negative control used was $0.9 \% \mathrm{NaCl}$.

\section{Biofilm formation}

The biofilm formation was developed as proposed by Pereira et al., ${ }^{18}$ with some modifications. The biofilms were grown on 50 sterilized acrylic resin (AC) discs (Clássico, São Paulo, Brazil), with a diameter of $6 \mathrm{~mm}$ each. The AC discs were placed in the first rows of 24-well plates (Costar Corning, New York, USA) containing $2 \mathrm{~mL}$ of sterile brain heart infusion (BHI) broth (Difco, Detroit, USA) supplemented with $5 \%$ sucrose and inoculated with $0.1 \mathrm{~mL}$ of each bacterial suspension. The AC discs were then incubated at $37^{\circ} \mathrm{C}$ for $48 \mathrm{~h}$. The media was not changed during the incubation period.

After $48 \mathrm{~h}$ of incubation, the AC discs contain- 
ing the biofilms were aseptically transferred to the second and third rows of the plate (24 wells) and washed twice with $2 \mathrm{~mL}$ of $0.9 \% \mathrm{NaCl}$ to remove loosely bound material.

\section{Susceptibility testing of biofilms}

The 50 AC discs with the biofilms formed after $48 \mathrm{~h}$ were placed in the fourth row of the plate ( 24 wells) and exposed for 5 minutes to $2 \mathrm{~mL}$ of the following solutions:

- C. aurantium EO ( $\mathrm{n}=10)$,

- C. limonum EO ( $\mathrm{n}=10)$,

- $0.2 \%$ CHX ( $\mathrm{n}=10)$,

- $1 \% \mathrm{NaOCl}(\mathrm{n}=10)$ and

- $0.9 \% \mathrm{NaCl}(\mathrm{n}=10)$.

Following the experimental periods, each biofilm was washed with $2 \mathrm{~mL}$ of $0.9 \% \mathrm{NaCl}$ to remove the substances tested. The discs were placed in tubes containing $10 \mathrm{~mL}$ of $0.9 \% \mathrm{NaCl}$ and sonicated (Sonoplus HD 2200, 50 W. Bandelin Eletronic, Berlin, Germany) for $30 \mathrm{~s}$ to disperse the biofilms. Biofilm suspensions were serially diluted in $0.9 \% \mathrm{NaCl}$ to produce dilutions of $10^{-1}$ to $10^{-5}$ times the original concentration. One hundred microliter aliquots of each dilution were seeded on agar (in duplicate):

- Sabouraud dextrose agar with $50 \mathrm{mg} / \mathrm{L}$ chloramphenicol (União Química, São Paulo, Brazil) for C. albicans,

- MacConkey agar for E. coli (HiMedia, Mumbai, India) and

- $m$-Enterococcus agar (Difco, Detroit, USA) for E. faecalis.

The number of colony-forming units per milliliter $(\mathrm{CFU} / \mathrm{mL})$ was determined after 48 hours of incubation. The results were $\log$-transformed $\left(\log _{10}\right)$ and analyzed by analysis of variance (ANOVA), and the Tukey test. A $p$ value of $<0.05$ indicated a statistically significant difference. The percentage of reduction was calculated using the $0.9 \% \mathrm{NaCl}$ results as a reference.

\section{Results}

This study aimed at evaluating the effects of $C$. limonum $\mathrm{EO}$ and $\mathrm{C}$. aurantium $\mathrm{EO}$, compared to other solutions of known antimicrobial activity, such as $0.2 \% \mathrm{CHX}$ and $1 \% \mathrm{NaOCl}$, on multi-species biofilms formed on acrylic resin by C. albicans, E. faecalis and E. coli reference strains. All tested solutions significantly decreased $(p<0.05)$ the viability of microorganism growth in multi-species biofilms formed on acrylic resin discs, as compared to the control group, which was treated only with $0.9 \%$ $\mathrm{NaCl}$. Figures 1, 2 and 3 show these results.

C. aurantium $\mathrm{EO}$ and $\mathrm{NaOCl}$ were the most effective solutions and inhibited the growth of all microorganisms in multi-species biofilms. C. limonum EO promoted a $100 \%$ reduction of C. albicans and E. coli, and $49.3 \%$ of E. faecalis biofilms.

We did not achieve the complete elimination of any microorganism with $0.2 \% \mathrm{CHX}$, which was less effective against $C$. albicans and E. coli, with a reduction of $68.8 \%$ and $86.7 \%$, respectively. However the reduction of E. faecalis with CHX (81.7\%) was greater than that using C. limonum EO.

\section{Discussion}

Microorganisms form biofilms as a means of defense and of facilitating physiologic processes. The layered organization of the biofilm protects the microorganisms from changes in $\mathrm{pH}$ and other antimicrobial insults. ${ }^{19}$ Furthermore, biofilm formation is

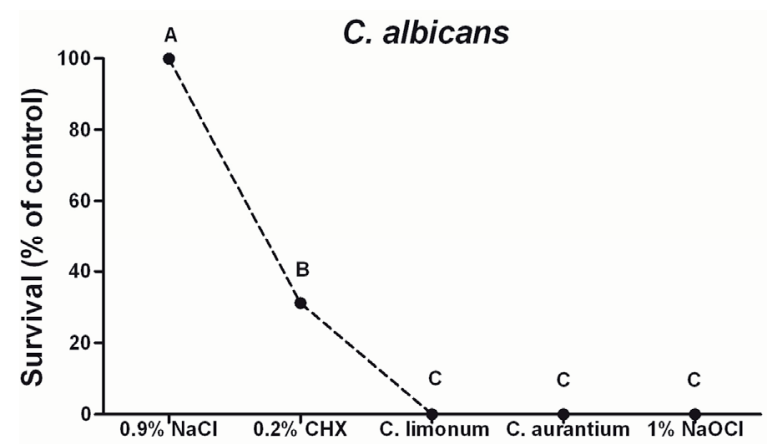

Figure 1 - Survival of C. albicans grown in multi-species biofilms formed in acrylic resin discs after treatment to determine the antimicrobial effects of $\mathrm{C}$. aurantium or C. limonum essential oils (EOs), compared to $0.2 \%$ chlorhexidine $(\mathrm{CHX})$ or $1 \%$ sodium hypochlorite $(\mathrm{NaOCl})$. Saline solution $(0.9 \% \mathrm{NaCl})$ treatment was used as a negative control. The results are given as a percentage of reduction, calculated with the $0.9 \% \mathrm{NaCl}$ results as a reference. Different letters indicate statistically significant differences, $p<0.05$ (ANOVA, Tukey's test). 


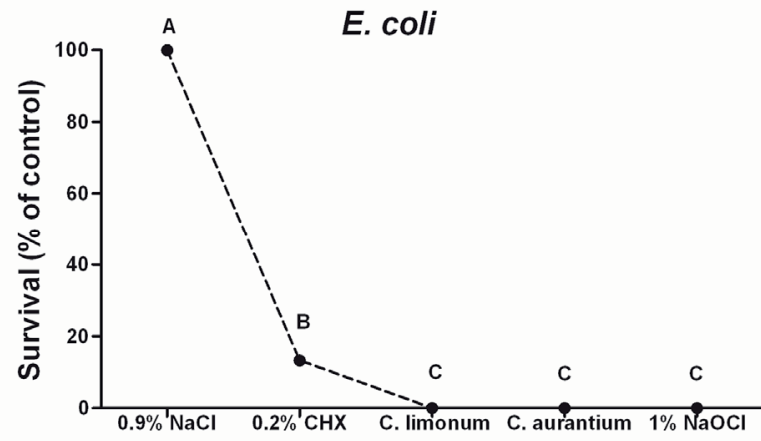

Figure 2 - Survival of E. coli grown in multi-species biofilms formed in acrylic resin discs after treatment to determine the antimicrobial effects of $\mathrm{C}$. aurantium or $\mathrm{C}$. limonum essential oils (EOs), compared to $0.2 \%$ chlorhexidine $(\mathrm{CHX})$ or $1 \%$ sodium hypochlorite $(\mathrm{NaOCl})$. Saline solution $(0.9 \% \mathrm{NaCl})$ treatment was used as a negative control. The results are given as a percentage of reduction, calculated with the $0.9 \%$ $\mathrm{NaCl}$ results as a reference. Different letters indicate statistically significant differences, $p<0.05$ (ANOVA, Tukey's test).

enhanced by coaggregation, which is the adhesion of two or more microorganism species. Biofilms can be up to 1000 times more resistant to antimicrobials than planktonic cells from the same species. ${ }^{20}$ These issues have called for alternative strategies to control biofilms, particularly in the context of treating oral disease.

This study aimed at evaluating the antimicrobial effects of C. limonum EO and C. aurantium EO, compared to other solutions of known antimicrobial effects, such as $0.2 \% \mathrm{CHX}$ and $1 \% \mathrm{NaOCl}$, on multi-species biofilms formed on acrylic resin by $C$. albicans, E. faecalis and E. coli reference strains. When we compared the EOs with these solutions, we found that the antimicrobial effects of the EOs were similar to that of $1 \% \mathrm{NaOCl}$, and that these effects promoted a $100 \%$ reduction of C. albicans and E. coli. C. aurantium $\mathrm{EO}$ and $1 \% \mathrm{NaOCl}$ also produced the complete elimination of E. faecalis in biofilms. These results were more favorable than those using $0.2 \% \mathrm{CHX}$. Currently, $\mathrm{CHX}$ is the safest and most efficient antimicrobial agent used to reduce microorganisms in the oral cavity. ${ }^{21,22}$ However, CHX is associated with a number of adverse effects, such as the formation of stains on teeth and dentures, dysgeusia, parotid enlargement, and desquamation

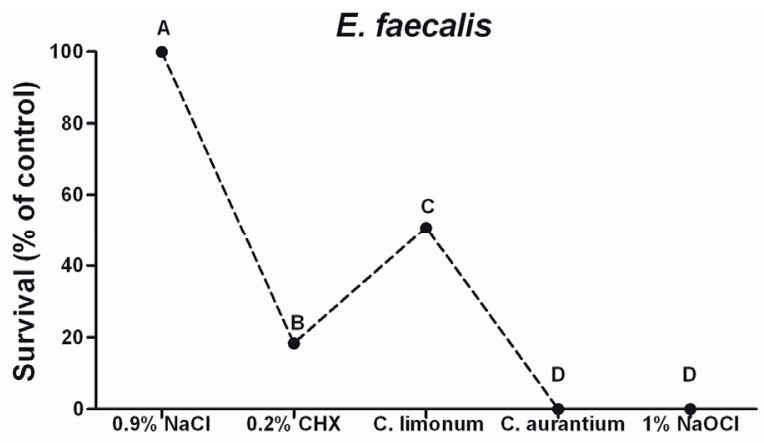

Figure 3 - Survival of E. faecalis grown in multi-species biofilms formed in acrylic resin discs after treatment to determine the antimicrobial effects of $\mathrm{C}$. aurantium or $\mathrm{C}$. limonum essential oils (EOs), compared to $0.2 \%$ chlorhexidine (CHX) or $1 \%$ sodium hypochlorite ( $\mathrm{NaOCl})$. Saline solution $(0.9 \%$ $\mathrm{NaCl}$ ) treatment was used as a negative control. The results are given as a percentage of reduction, calculated with the $0.9 \% \mathrm{NaCl}$ results as a reference. Different letters indicate statistically significant differences, $p<0.05$ (ANOVA, Tukey's test).

of the oral mucosa. ${ }^{21} \mathrm{NaOCl}$ also provides antimicrobial activity against microorganisms in the oral cavity, and has been used as an endodontic irrigant. However, it is cytotoxic when injected into the periapical tissues, where it may cause severe inflammatory reactions. ${ }^{23}$ These factors have prompted the search for other antimicrobial agents.

In this study, the viability of C. albicans in multispecies biofilms was directly affected by C. limonum $\mathrm{EO}$ and C. aurantium EO, which eliminated the yeast in the concentrations that were used. Other EOs from various Citrus species (C. limonum, C. paradisi, C. bergamia, C. sinensis and C. reticulata) showed antimicrobial activity on planktonic cultures of grampositive and gram-negative bacteria, including E. coli and yeasts such as C. albicans. ${ }^{12}$ Oral candidiasis is caused by Candida yeasts, which are present in the oral cavities of approximately half of all healthy individuals. ${ }^{24} \mathrm{C}$. albicans is the most virulent and prevalent species, isolated in up to $50 \%$ of cases of oral candidiasis; ${ }^{25}$ it colonizes the oral surface, proliferates and causes damage through the expression of its virulence factors, such as adherence to host cells, morphological transition, biofilm formation, hydrophobicity and secretion of hydrolytic enzymes. ${ }^{26}$

C. limonum $\mathrm{EO}$ and C.aurantium $\mathrm{EO}$ promoted 
a $100 \%$ reduction of $E$. coli in multi-species biofilms in this study. A study conducted by Soković et al. ${ }^{13}$ analyzed the activities of various EOs, including C. aurantium and C. limonum, against pathogenic microorganisms, and observed growth inhibition of E. coli planktonic cultures in disc diffusion and microdilution tests. Ashok-Kumar et al. ${ }^{14}$ evaluated the antibacterial activities of five different solvent extracts (ethyl acetate, acetone, ethanol, petroleum ether and water) prepared from the peels of two citrus fruits (C. sinensis and C. limonum) against five pathogenic bacteria: Staphylococcus aureus, Bacillus subtilis, E. coli, Klebsiella pneumoniae and Salmonella typhi. These authors observed high antibacterial activity against these bacteria in planktonic cultures, and concluded that extracts of $C$. sinensis and C. limonum can be as potent as methicillin and penicillin. In the oral cavity, E. coli is considered a transient colonizer, and is more prevalent in the mouth as a successional community during antibiotic therapy, and also in immunocompromised and hospitalized patients. Among the virulence factors of E. coli, the endotoxin produced by this bacterium plays a role in early and perpetuation of periapical inflammatory lesions and bone resorption.

In this study, C. limonum EO reduced the viability of E. faecalis in multi-species biofilms by $49.3 \%$, whereas C. aurantium EO eliminated this microorganism. Laird et al. ${ }^{16}$ also achieved the complete elimination of E. faecalis biofilms established on stainless steel surfaces with a vaporized blend of citrus EO (Citri-V ${ }^{\mathrm{TM}}$, orange: bergamot, $1: 1 \mathrm{v} / \mathrm{v}$ ). The results of previous studies investigating the effects of citrus vapor on Enterococcus sp. suggested that it acts on the cell membrane, insofar as treated cells show a loss of membrane integrity and an increase in cell permeability compared to untreated cells, with a loss of membrane potential and a reduction in intracellular ATP. ${ }^{27}$ In the oral cavity, E. faecalis is commonly isolated in

\section{References}

1. Costerton JW, Stewart PS, Greenberg EP. Bacterial biofilms: a common cause of persistent infections. Science. 1999 May $21 ; 284(5418): 1318-22$. root canal systems as an outcome of failed endodontic treatment, on account of its ability to adhere to dentin, invade dentinal tubules and form biofilms; this may contribute to bacterial resistance and persistence after intracanal antimicrobial procedures. ${ }^{5}$

The mechanisms by which EOs can inhibit microorganisms involve different modes of action and may in part be an outcome of microorganism hydrophobicity. As a result, the oils are partitioned into the lipid bilayer of the cell membrane, affecting the respiratory chain and leading to the leakage of vital cell contents. ${ }^{28}$ The impairment of bacterial enzyme systems may also be a potential mechanism of action. Various components of EOs can permeabilize the cell membrane, increasing the penetration of antibiotics. Interference with bacterial enzyme systems may be another potential mechanism of action..$^{29}$ Chemical analyses have shown that limonene is the most abundant chemical component of C. aurantium and C. limonum EOs, at $90 \%$ and $59.7 \%$, respectively. Limonene was tested separately for antimicrobial activity and was confirmed to have bactericidal activity against gram-positive and gram-negative bacteria, including E. coli. ${ }^{13}$

\section{Conclusion}

The current results indicate that EOs are effective in controlling multi-species biofilms, and that the microbial reductions achieved by EOs are not only similar to those of $\mathrm{NaOCl}$, but even higher than those achieved by CHX, in some cases.

\section{Acknowledgements}

This work was supported by the Fundação de Amparo à Pesquisa do Estado de São Paulo (FAPESP), Brazil (Grant 09/52048-1).

The author Cristiane A. Pereira is grateful to FAPESP for the provided scholarship (Process 2010/00879-4).

2. Seneviratne CJ, Jin L, Samaranayake LP. Biofilm lifestyle of Candida: a mini review. Oral Dis. 2008 Oct;14(7):582-90.

3. Oliveira LD, Carvalho CA, Carvalho AS, Alves JS, Valera $\mathrm{MC}$, Jorge AO. Efficacy of endodontic treatment for endotoxin 
reduction in primarily infected root canals and evaluation of cytotoxic effects. J Endod. 2012 Aug;38(8):1053-7.

4. Rôças IN, Siqueira JF, Santos KRN. Association of Enterococcus faecalis with different forms of periradicular diseases. $\mathrm{J}$ Endod. 2004 May;30(5):315-20

5. Sedgley CM, Lennan SL, Appelbe OK. Survival of Enterococcus faecalis in root canals ex vivo. Int Endod J. 2005 Oct;38(10):735-42.

6. Barnett ML. The role of therapeutic antimicrobial mouthrinses in clinical practice: control of supragingival plaque and gingivitis. J Am Dent Assoc. 2003 Jun;134(6):699-704.

7. Davies D. Understanding biofilm resistance to antibacterial agents. Nat Rev Drug Discov. 2003 Feb;2(2):114-22.

8. Chandrasekaran M, Venkatesalu V. Antibacterial and antifungal activity of Syzygium jambolanum seeds. J Ethnopharmacol. 2004 Mar;91(1):105-8.

9. Eloff JN. Wich extractant should be used for the screening and isolation of antimicrobial components from plants? J Ethnopharmacol. 1998 Feb;60(1):1-8.

10. Ahmad MM, Rehman S, Iqbal Z, Anjum FM, Sultan JI. Genetic variability to essential oil composition in four Citrus fruit species. Pak J Bot. 2006 Feb;38(2):319-24.

11. Adedejl GB, Fagade OE, Oyelade AA. Prevalence of Pseudomonas aeruginosa in clinical samples and its sensitivity to Citrus Extracts. Afr J Biomed Res. 2007 May;10(2):183-7.

12. Kirbaşlar GF, Tavman A, Dülger B, Türker G. Antimicrobial activity of Turkish citrus peel oils. Pak J Bot. 2009;41(6):320712.

13. Soković M, Glamočlija J, Marin PD, Brkić D, van Griensven LJLD. Antibacterial effects of the essential oils of commonly consumed medicinal herbs using an in vitro model. Molecules. 2010 Oct;15(11):7532-46.

14. Ashok-Kumar K, Narayani M, Subanthini A, Jayakumar M. Antimicrobial activity and phytochemical analysis of Citrus fruit peels - utilization of fruit waste. Int J Eng Sci Technol (IJEST). 2011 Jun;3(6):5414-21.

15. Saviuc C, Dascălu L, Chifiriuc MC, Rădulescu V, Oprea E, Popa M, et al. The inhibitory activity of pomelo essential oil on the bacterial biofilms development on soft contact lenses. Roum Arch Microbiol Immunol. 2010 Jul-Sep;69(3):145-52.

16. Laird K, Armitage D, Phillips C. Reduction of surface contamination and biofilms of Enterococcus sp. and Staphylococcus aureus using a citrus-based vapour. J Hosp Infect. 2012 Jan;80(1):61-6.

17. Pereira CA, da Costa AC, Machado AK, Beltrame Júnior M, Zöllner MS, Junqueira JC, et al. Enzymatic activity, sensitivity to antifungal drugs and Baccharis dracunculifolia essential oil by Candida strains isolated from the oral cavities of breastfeeding infants and in their mothers' mouths and nipples. Mycopathologia. 2011 Feb;171(2):103-9.

18. Pereira CA, Romeiro RL, Costa AC, Machado AK, Junqueira JC, Jorge AO. Susceptibility of Candida albicans, Staphylococcus aureus, and Streptococcus mutans biofilms to photodynamic inactivation: an in vitro study. Lasers Med Sci. 2011 May;26(3):341-8.

19. Chavez de Paz LE. Redefining the persistent infection in root canals: possible role of biofilm communities. J Endod. 2007 Jun;33(6):652-62.

20. Mah TF, Pitts B, Pellock B, Walker GC, Stewart PS, O’Toole GA. A genetic basis for Pseudomonas aeruginosa biofilm antibiotic resistance. Nature. 2003 Nov 20;426(6964):306-10.

21. Groppo FC, Ramacciato JC, Simões RP, Flório FM, Sartoratto A. Antimicrobial activity of garlic, tea tree oil, and chlorhexidine against oral microorganisms. Int Dent J. 2002 Dec;52(6):433-7.

22. Meiller TF, Kelley JI, Jabra-Rizk MA, DePaola LG, Baqui AAM, Falkler WA Jr. In vitro studies of the efficacy of antimicrobials against fungi. Oral Surg Oral Med Oral Pathol Oral Radiol Endod. 2001 Jun;91(6):663-70.

23. Gernhardt CR, Eppendorf K, Kozlowski A, Brandt M. Toxicity of concentrated sodium hypochlorite used as an endodontic irrigant. Int Endod J. 2004 Apr;37(4):272-80.

24. Martins CAP, Koga-Ito CY, Jorge AOC. Presence of Staphylococcus spp. and Candida spp. in the human oral cavity. Braz J Microbiol. 2002 Jul-Sep;33(3):236-40.

25. Delgado ACD, Pedro RJ, Aoki FH, Resende MR, Trabasso P, Colombo AL, et al. Clinical and microbiological assessment of patients with a long-term diagnosis of human immunodeficiency virus infection and Candida oral colonization. Clin Microbiol Infect. 2009 Apr;15(4):364-71.

26. Mishra NN, Prasad T, Sharma N, Payasi A, Prasad R, Gupta DK, et al. Pathogenicity and drug resistance in Candida albicans and other yeast species. Acta Microbiol Immunol Hung. 2007 Sep;54(3):201-35.

27. Fisher K, Phillips C. The mechanism of action of a citrus oil blend against Enterococcus faecium and Enterococcus faecalis. J Appl Microbiol. 2009 Apr;106(4):1343-9.

28. Burt S. Essential oils: their antibacterial properties and potential applications in foods - a review. Int J Food Microbiol. 2004 Aug 1;94(3):223-53.

29. Wendakoon C, Sakaguchi M. Inhibition of amino acid decarboxylase activity of Enterobacter aerogenes by active components in spices. J Food Prot. 1995 Mar;58(3):280-3. 